\title{
Determining optimal location of static VAR compensator by means of genetic algorithm
}

\begin{abstract}
The purpose of this paper is to study a practical and accurate heuristic method known as genetic algorithm (GA) which is used to find the optimal location of Static Var Compensator (SVC) and its appropriate size and setting. This method is employed to optimize the stability of power system by means of maximizing distance to collapse point. The continuation power flow (CPF) method is employed to determine the collapse point and critical area of power system. Moreover, the total real power loss of system is minimized by this algorithm. Modeling and simulation is performed on IEEE 14, 57 and 118 bus test system. The obtained result implements the effectiveness of this method to find the best location, appropriate size and setting of this element to the desired targets.
\end{abstract}

Keyword: Voltage collapse; FACTS devices; SVC; CPF; Genetic algorithm 